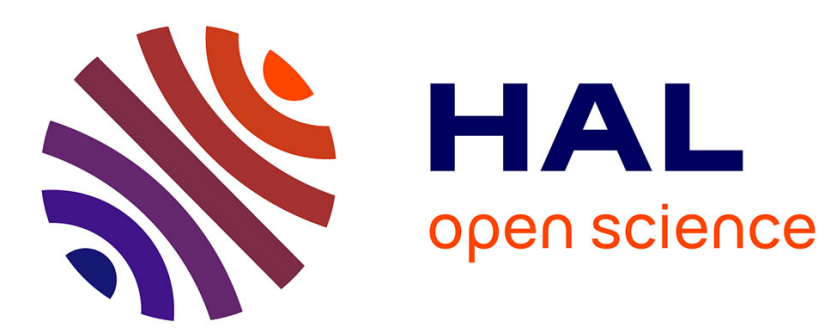

\title{
Se nourrir à Hanoi : Les recompositions du système alimentaire d'une ville émergente
}

Gwenn Pulliat

\section{To cite this version:}

Gwenn Pulliat. Se nourrir à Hanoi : Les recompositions du système alimentaire d'une ville émergente. EchoGéo, 2012, 21, 10.4000/echogeo.13205 . hal-01453471

\section{HAL Id: hal-01453471 \\ https://hal.science/hal-01453471}

Submitted on 2 Feb 2017

HAL is a multi-disciplinary open access archive for the deposit and dissemination of scientific research documents, whether they are published or not. The documents may come from teaching and research institutions in France or abroad, or from public or private research centers.
L'archive ouverte pluridisciplinaire HAL, est destinée au dépôt et à la diffusion de documents scientifiques de niveau recherche, publiés ou non, émanant des établissements d'enseignement et de recherche français ou étrangers, des laboratoires publics ou privés. 


\title{
EchoGéo
}

numéro 21 | 2012 :

juillet 2012/septembre 2012

Sur le Champ/Sur le Terrain

Pays émergents

\section{Se nourrir à Hanoi : les recompositions du système alimentaire d'une ville émergente}

\author{
GWENN PULLIAT
}

\section{Résumés}

\section{Français English}

Si la capitale vietnamienne profite au premier chef des transformations liées au décollage économique du Vietnam depuis les années 1990, l'analyse de la filière alimentaire permet de mettre au jour la façon dont les pratiques quotidiennes des habitants sont marquées par les mutations de cette ville émergente. Elle révèle une image contrastée de la ville : entre ouverture aux marchés internationaux et persistance d'une production agricole familiale, entre modernisation des marchés et persistance de la vente de rue, entre transition alimentaire et persistance de l'insécurité. L'exemple de l'alimentation donne à voir comment les mutations de Hanoi creusent les disparités entre les groupes sociaux.

If the Vietnamese capitale city primiraly benefits from the shifts in which the economic takeoff of Vietnam has resulted since early 1990, analyzing the food system sheds light on the way that mutations of this emerging city impact people's food practices. It reveals a two-tiered city: Hanoi is more and more engaged into international markets but familial farming is persistent; markets are involved in a modernizing process but street vending is still important; food transition has begun but food insecurity remains. The example of food shows how mutations of Hanoi deepen social inequalities.

\section{Entrées d'index}

Mots-clés : Hanoi, inégalité sociale, sécurité alimentaire, transition alimentaire, ville émergente

Keyword : ermerging city, food security, food transition, Hanoi, social inequalty

\section{Texte intégral}




\section{Introduction} devenu le deuxième exportateur mondial de riz ! » : cette exclamation énoncée par un vice-ministre vietnamien à la Planification illustre l'image d'abondance associée au statut de grand producteur de denrées alimentaires, au premier rang desquelles le riz, qui contraste singulièrement avec les pénuries qui prévalaient au Vietnam avant le Đổi Mó́i ${ }^{1}$. Elle illustre aussi cette tendance des autorités vietnamiennes à considérer l'autosuffisance alimentaire à l'échelle nationale comme un garant de la sécurité alimentaire des ménages - à l'exception de régions montagneuses pauvres et reculées pour lesquelles des programmes de développement agricole et de réduction de la pauvreté sont spécifiquement développés. Pourtant, cette posture macro-économique centrée sur un seul aliment occulte les individus, leurs pratiques quotidiennes, leurs difficultés, parfois, à acquérir les denrées qui sont effectivement disponibles sur le marché mais trop onéreuses pour leurs bourses, surtout quand d'autres frais se présentent. Certaines personnes peuvent alors être privées d'accès à une alimentation suffisante, saine, de bonne qualité nutritionnelle et correspondant à ses préférences - ce qui caractérise la sécurité alimentaire selon la définition adoptée par le Sommet Mondial de l'Alimentation en 1996 (Bricas et al., 2003).

2 On voit ainsi se dessiner une image contrastée de la capitale vietnamienne. D'un côté, celle de la capitale d'un pays qui connaît une croissance économique soutenue depuis près de trois décennies ${ }^{2}$. La politique du Đổi Mói a promu la modernisation de l'économie du pays en s'appuyant sur l'industrialisation et l'ouverture à l'international. Ces transformations économiques se sont accompagnées d'un mouvement d'urbanisation soutenu (le taux de population urbaine est passé de $19 \%$ en 1989 à $30 \%$ aujourd'hui) et d'une amélioration remarquable des conditions de vie des habitants. C'est en priorité les deux métropoles, Ho Chi Minh Ville et Hanoi, qui ont bénéficié des politiques de réformes. Leur dynamisme économique, soutenu par des plans de développement promus par l'État, assure leur attractivité pour les investisseurs internationaux. Cela incite à leur appliquer le qualificatif d' « émergentes » : utilisé d'abord par Antoine van Agtamael en 1981 pour désigner le fort potentiel économique de marchés, il a ensuite été appliqué à des pays caractérisés par leur décollage économique, leur insertion dans l'économie mondiale et une attractivité forte pour les investisseurs étrangers (Jaffrelot, 2008). $\mathrm{Au}$ Viet Nam, ce sont les deux métropoles qui polarisent ces dynamiques économiques et en sont le support spatial - ce qui nous incite à leur attribuer l'épithète, plutôt qu'au pays dans son entier. Ainsi, Hanoi apparaît comme une capitale émergente dans un pays désormais classé parmi les économies à revenus moyens (données Banque Mondiale, 2011).

Mais d'un autre côté, on observe, en même temps, un accroissement des inégalités socioéconomiques (Gironde, 2009) dont témoigne le développement, à Hanoi, d'une population précaire, occupant des emplois à statut instable et aux revenus fluctuants. Il ne s'agit pas ici de débattre sur les mesures de la pauvreté urbaine et des inégalités, mais de s'intéresser aux pratiques alimentaires quotidiennes de cette population qui ne participe pas directement à ce mouvement de modernisation, et qui se trouve vulnérable aux chocs économiques qui se présentent. L'importance de l'alimentation dans le budget (49\% en moyenne en ville en 2010 selon le GSO (VHLSS, 2010), et même 65,4 \% dans le premier quintile de revenus) en fait la première variable d'ajustement des dépenses quotidiennes.

4 Ces personnes vivent dans ce contexte en mutation rapide, dans une ville qui connaît depuis les années 1980 une forte croissance démographique - 4\% par an en moyenne (Castiglioni et al., 2006) et une rapide extension de sa tache urbaine. Elles ne sont aucunement passives : les choix économiques des ménages, dont les 
pratiques alimentaires sont un élément, sont élaborés en fonction des ressources, des opportunités, mais aussi des difficultés qu'ils rencontrent (Maxwell, 1996 ; Maxwell, Smith, 1992), et leurs pratiques impriment leurs marques sur l'espace et son organisation. On voit là se dessiner un hiatus entre les projets d'une ville moderne promue par les autorités, devant être l'image d'un pays attractif, et l'espace vécu et utilisé au quotidien par les citadins (riches comme pauvres).

Quelles sont alors les caractéristiques de la filière alimentaire dans une ville émergente ? Le processus d'émergence semble, dans le domaine alimentaire, s'exprimer par plusieurs phénomènes :

- Une transition alimentaire (Charvet, 2009) particulièrement rapide

- Cette transition est cependant inégale parmi les différents groupes sociaux et on observe une disparité croissante dans les modes de consommation selon les revenus (Landy, 2008)

- Cela se traduit alors par la coexistence de troubles liés à la suralimentation et de maladies liées à la malnutrition par carences (Calandre, 2006)

- Enfin, la question sanitaire s'impose progressivement comme une préoccupation importante tant pour les consommateurs que pour les autorités publiques.

$6 \quad$ Nous nous attacherons ici à voir quelles sont les structures et les recompositions du système alimentaire à Hanoi - c'est-à-dire la façon dont s'organisent la production, la distribution et la consommation des aliments (Malassis, 1993). Comment la forte croissance économique qui caractérise les pays émergents, à l'origine d'un intense mouvement d'urbanisation et de profondes transformations du tissu économique, influence-t-elle les pratiques quotidiennes des habitants ? Nous faisons l'hypothèse que l'émergence économique se traduit par des disparités croissantes entre les différents groupes sociaux et nous tenterons de voir comment cela se traduit dans le système alimentaire urbain. Nous nous intéresserons en particulier aux recompositions en cours dans l'approvisionnement de la ville, dans la distribution et dans les modes de consommation, dans le cadre des transformations rapides que connaît la capitale du Vietnam.

Le présent article se fonde les premiers résultats de notre enquête de terrain menée à Hanoi de l'automne 2010 au printemps 2012. Cette enquête a pris la forme d'une centaine d'entretiens qualitatifs, dans trois quartiers différents de la capitale : un quartier central, un ancien village désormais intégré à la ville et un village périurbain situé dans une zone faisant l'objet de plusieurs grands projets urbains (autoroute, zone industrielle, nouvelle zone d'habitation). Les entretiens semi-directifs avaient pour objet de restituer les trajectoires de vie des enquêtés, tout en insistant particulièrement sur les habitudes quotidiennes, à commencer par les pratiques alimentaires. Il s'agissait de s'intéresser particulièrement à la population urbaine défavorisée, caractérisée soit par un faible niveau de revenus (personnes ayant le statut de "foyer pauvre », avec un revenu inférieur au seuil de pauvreté officiel), soit par des conditions de vie particulièrement difficiles (logement insalubre, espaces de vie réduits et partagés etc.), soit par des formes d'emploi précaires avec des revenus fluctuants (cas de nombreux vendeurs de rue, de porteurs dans les marchés de nuit par exemple). Nos enquêtes ne présentent donc aucun caractère représentatif de l'ensemble de la population de Hanoi, mais illustrent une grande diversité de situations où l'on voit apparaître les différentes stratégies quotidiennes des acteurs pour faire face aux difficultés qu'ils rencontrent.

\section{L'offre alimentaire : modernisation agricole, libéralisation des marchés et}




\section{mutation de la production}

Le système alimentaire urbain débute là où sont produites les denrées qui approvisionnent la ville (Malassis, 1993). Ces réseaux d'approvisionnement se déploient à plusieurs niveaux et en fonction du type de produit considéré. A l'échelle du pays d'abord, la capitale bénéficie du développement de la production agricole nationale et de l'intégration aux marchés mondiaux. A l'échelle régionale ensuite, les rapports entre la ville grandissante et les régions limitrophes révèlent une tension entre un usage agricole de l'espace et le développement industriel et urbain. Enfin, à l'échelle de la ville on observe une dynamique de mise en valeur agricole des espaces urbains et périurbains encore libres, qui trouve sa source dans des motifs très variés.

\section{Une intégration aux marchés internationaux accompagnant une hausse remarquable de la production}

Après des années de rationnement alimentaire, la décollectivisation des terres entreprise dans les années 1980, par laquelle les foyers ruraux ont obtenu une parcelle de terrain et la possibilité de choisir, dans une certaine mesure, les cultures qu'ils y développeraient, a permis un renouveau spectaculaire de la production agricole (Dao The Tuan, 2002). Avec la modernisation de l'agriculture, et en particulier l'usage d'engrais chimiques ${ }^{3}$ et autres produits phytosanitaires, les rendements ont fortement augmenté et le Vietnam est devenu exportateur de denrées alimentaires (Fanchette, Dao The Anh, 2008). Selon les données de la FAO, il est ainsi en 2009 le deuxième exportateur mondial de riz ${ }^{4}$ et assure 10 à $15 \%$ des volumes échangés sur les marchés mondiaux. Il est également le deuxième exportateur de café, le premier pour les noix de cajou, et conserve une bonne place pour les produits de la mer, le poivre etc. En valeur, les exportations agricoles (tous aliments confondus) représentent plus du quart de la production agricole nationale (FAO, 2005). Au total, alors qu'en 1988 le pays devait importer entre autres du riz, de la farine de blé, du sucre, la production nationale est devenue suffisante pour approvisionner le marché au cours des années 2000, et l'État a commencé à commercialiser la production excédentaire sur les marchés internationaux (Cao, Tran, 2005).

La modernisation de l'économie consécutive au Đổi Mói s'est en effet fondée sur l'ouverture internationale. En 1994, les États-Unis ont mis un terme à l'embargo contre le Vietnam : cela a constitué la première étape de la réintégration du pays dans le commerce international. Le Vietnam est ensuite devenu, en 1997, membre de l'association économique régionale, l'ASEAN, puis a prolongé ses réformes économiques et douanières afin d'entrer dans l'OMC, dont il est devenu le $150^{\mathrm{e}}$ membre en 2007 (Cling et al., 2008). Cette intégration internationale, et en particulier les accords de libre-échange au sein de l'ASEAN et avec la Chine (effectifs depuis 2010), assure le développement du commerce extérieur et facilite l'entrée sur le marché vietnamien de denrées alimentaires étrangères. Ainsi, la Chine est désormais le premier pays d'importation de biens pour le Vietnam (en valeurs, données GSO 2012), et se présente comme un pourvoyeur important de denrées alimentaires, surtout pour le nord du pays : Sautier et al. (2011) observent lors de leur enquête sur les marchés de gros de Hanoi qu'en été5, un tiers des tomates et la totalité des choux (deux des cinq légumes étudiés, parmi les plus consommés dans la capitale) viennent de Chine. Une partie importante des fruits vendus à Hanoi sont également importés de Chine, mais nous n'avons pas de données précises les concernant. 
De la sorte, l'augmentation remarquable des rendements agricoles et l'intégration aux marchés mondiaux, dont les marchés des métropoles sont les premiers bénéficiaires, assurent un approvisionnement abondant en denrées alimentaires diversifiées : c'est cette image macroéconomique qui fonde celle d'une capitale dénuée d'insécurité alimentaire. L'État, qui contrôle étroitement les exportations, donne la priorité au marché intérieur et n'a pas hésité, en 2008, à interdire pendant plusieurs mois toute exportation de riz afin d'éviter une spéculation sur les marchés internationaux au détriment du marché intérieur, et d'assurer le maintien d'un approvisionnement national tout en limitant l'augmentation des prix. Le pays pourvoit à l'essentiel de ses besoins en produits de base (riz, légumes-feuilles qui se conservent de toute façon très mal, porc...), même si on observe une concurrence croissante avec les productions des pays voisins (à commencer par la Chine), et qu'il dépend des importations pour certains produits de consommation moins courante, quoiqu'en développement, comme les produits laitiers.

\section{Hanoi et son hinterland : tensions entre production alimentaire et urbanisation de l'espace}

Si le marché alimentaire de la capitale distribue des denrées provenant de tout le pays, voire de l'étranger, il constitue d'abord un débouché pour les productions issues de la zone rurale du Grand Hanoi ${ }^{6}$ et des provinces limitrophes de la capitale. La proximité du marché urbain, accrue par l'amélioration des infrastructures de transport, suscite une production de denrées à forte valeur ajoutée : maraîchage, élevage de volailles et de porcs. A titre d'exemple, Leo Van de Berg (2003) observa qu'entre 1991 et 2001, le nombre de porcs dans la province de Hanoi a crû de 50 \%, puis encore de $35 \%$ entre 2001 et 2011 (Sautier et al., 2011). La croissance de la population comme des revenus urbains induit une demande accrue, surtout en produits frais (Sautier et al., 2011) : Georges Rossi (2002) note ainsi que, durant les années 1990, la demande de légumes à Hanoi a augmenté de $15 \%$ par an. L'hinterland de la capitale joue donc un rôle d'espace de production agricole au profit de la ville, en particulier pour les produits périssables et fragiles (légumes, fruits, viande) : il s'agit là de l'influence classique du développement urbain sur l'espace environnant, dont les productions se transforment pour répondre à la demande urbaine et gagnent en valeur ajoutée.

Mais le développement de Hanoi renforce la concurrence entre une utilisation agricole de l'espace et l'urbanisation. En réponse à la croissance économique et démographique soutenue, Hanoi s'étend rapidement et de nombreux espaces agricoles sont convertis en terrains bâtis - même si nous ne disposons pas de données quantifiées de ces conversions (Sautier et al., 2011). Les projets de zones industrielles et de nouvelles zones urbaines sont nombreux. Cette croissance de la tache urbaine, répondant aux projets de développement promus par l'État qui entend donner à la capitale une réelle envergure internationale, interroge en même temps les autorités : l'Assemblée Nationale a voté en 2009 un plan d'aménagement foncier qui prévoit la préservation, à l'échelle nationale, de près de 4 millions d'hectares de rizières afin de protéger l'approvisionnement du marché vietnamien. Dans le delta du Fleuve Rouge, principal pourvoyeur de riz du nord du pays, on voit ainsi une tension se créer entre la volonté d'établir une capitale d'envergure internationale d'une part, et ce souci de préserver une production indispensable pour approvisionner le marché urbain d'autre part.

Illustration 1 - Extension de la tache urbaine dans la province de Hanoi 


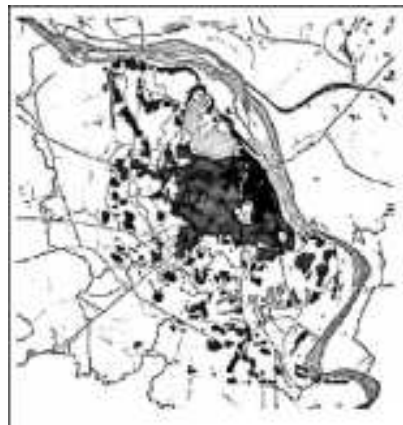

1983

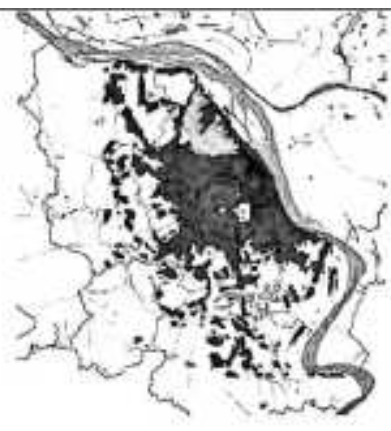

1996

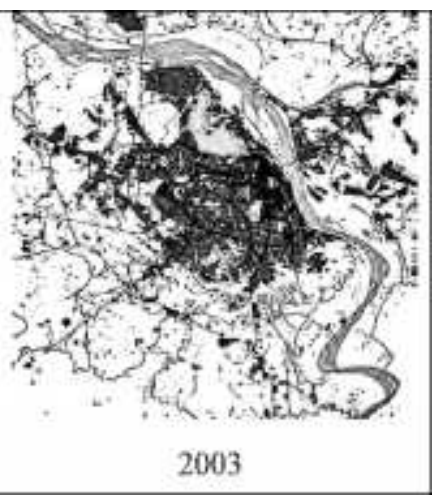

2003

Le développement urbain de Hanoi a conduit à un étalement de la ville vers l'ouest principalement, surtout le long des principaux axes de transport. La ville a progressivement absorbé les anciens villages périurbains et leurs zones agricoles, qui constituent désormais des quartiers de la capitale.

Source : Pham Thai Son (2010), d'après JICA (2007).

\section{Illustration 2 - Un village périurbain en transition - An Khánh}

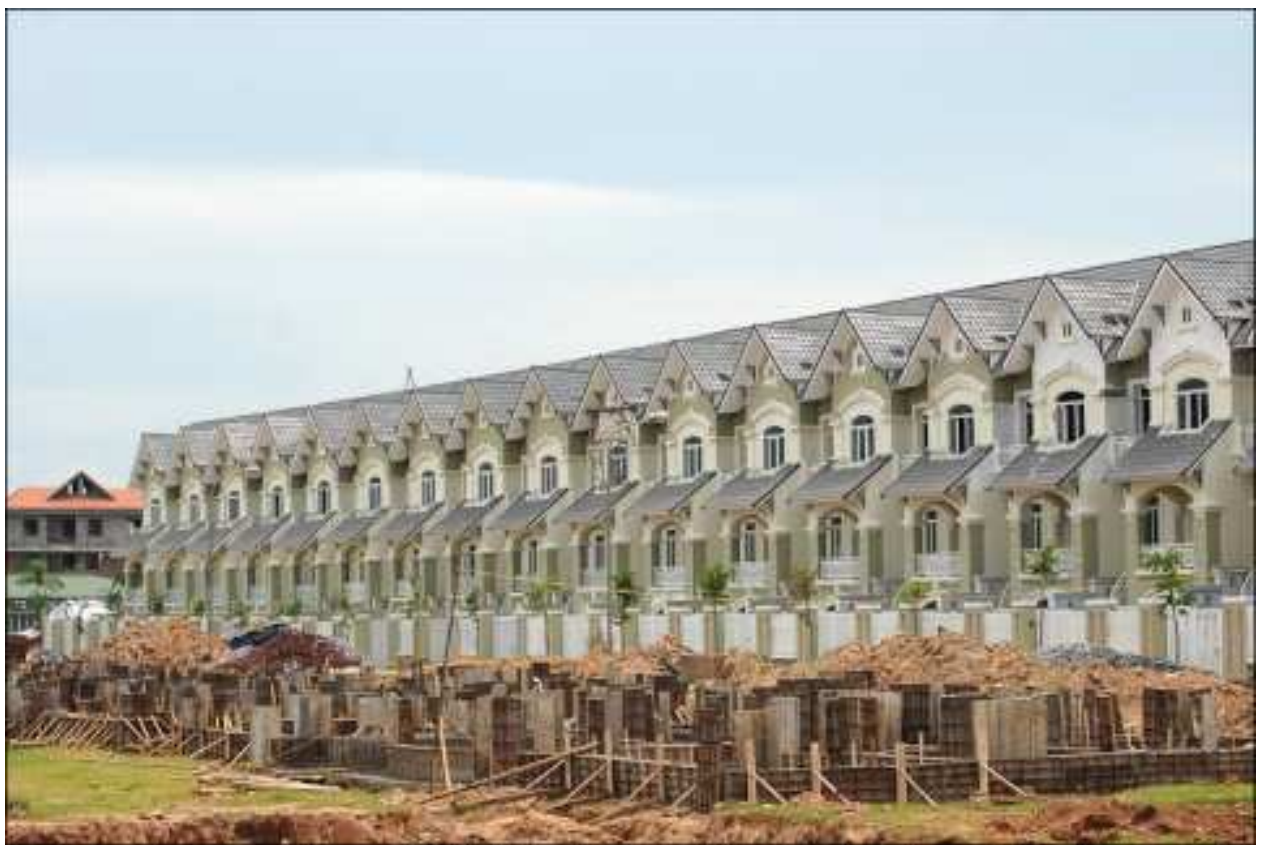

Le projet de ville nouvelle « Splendora » et « An Khánh Sud » le long de l'autoroute de l'Ouest s'est accompagné de l'expropriation de toutes les terres agricoles de la commune, afin de construire de nouvelles zones d'habitat et un pôle économique. Ici, les rangées de maisons encore en construction contrastent fortement avec l'habitat préexistant, qu'il s'agisse des anciennes maisons à un niveau autour d'une cour, des grosses maisons individuelles ou des maisons-tubes plébiscitées par les habitants qui reconstruisent leur logement.

Auteur : G. Pulliat, 2011.

\section{Une persistance de la petite production familiale}

L'extension de la tache urbaine autour de la capitale et, à échelle plus large, le développement urbain des régions limitrophes sont à l'origine d'une mutation classique mais ici particulièrement rapide de ces espaces périphériques. Ces transformations interviennent dans un espace très densément peuplé (de l'ordre de 1 ooo habitants par kilomètre carré pour l'ensemble du delta du Fleuve Rouge, environ 2000 pour la province de Hanoi) où les ressources économiques combinent le plus souvent activité agricole et artisanat, emploi industriel ou dans les services (Gironde, 2009). En effet, toutes les familles ont obtenu des terres agricoles en 1993 avec l'achèvement de la décollectivisation, et en 2005 un rapport de la Banque Mondiale (Poverty Task Force, 2005) établissait que 78 \% des ménages du delta du Fleuve Rouge déclaraient exercer une activité agricole. Néanmoins, seuls $17 \%$ d'entre eux en tiraient l'intégralité de leurs revenus. La diversification des activités 
avait débuté dès avant le Đổi Mói (Gironde, 2009) et s'est accentuée d'autant plus rapidement que les terrains attribués étaient de très petite taille : en général, dans le delta, chaque personne née avant 1993 a obtenu $360 \mathrm{~m}^{2}$. Selon tous nos enquêtés, cette surface assure la consommation de riz d'une personne sur l'année, mais ne permet pas de nourrir une famille avec des enfants nés après 1993 (donc sans terre supplémentaire), et encore moins de financer les autres dépenses de la vie quotidienne.

L'articulation de plusieurs types de ressources - agricoles, artisanales, industrielles, dans les services - se présente alors comme un facteur d'adaptation des familles dans un contexte économique en mutation rapide. Ainsi, notre enquête révèle un fort attachement à cette petite production familiale, qui se déploie sur toutes les terres disponibles, y compris dans les quartiers urbains périphériques : dans nos trois quartiers, les enquêtés qui disposent de terres cultivées ${ }^{7}$ y associent un fort sentiment de sécurité, car cela assure un approvisionnement alimentaire indépendant des fluctuations des revenus, permettant de compléter les courses quotidiennes et de réduire le budget alimentaire. Ce complément aux autres activités économiques se trouve mobilisé dès qu'un espace est disponible : ainsi, à la suite de la construction de barrages en amont de la capitale, les berges du Fleuve Rouge ne sont plus inondées que très occasionnellement, et l'espace libéré a aussitôt été mis à profit. Dans notre quartier d'étude au centre de Hanoi, les ordures amoncelées sur la berge sont progressivement remplacées par des jardins potagers et des vergers.

\section{Illustrations 3a et 3b. Quartier de Phúc Tân, Hanoi : cultures sur la berge du Fleuve} Rouge : chou-rave, laitue, liserons d'eau, chayottes, plantes aromatiques

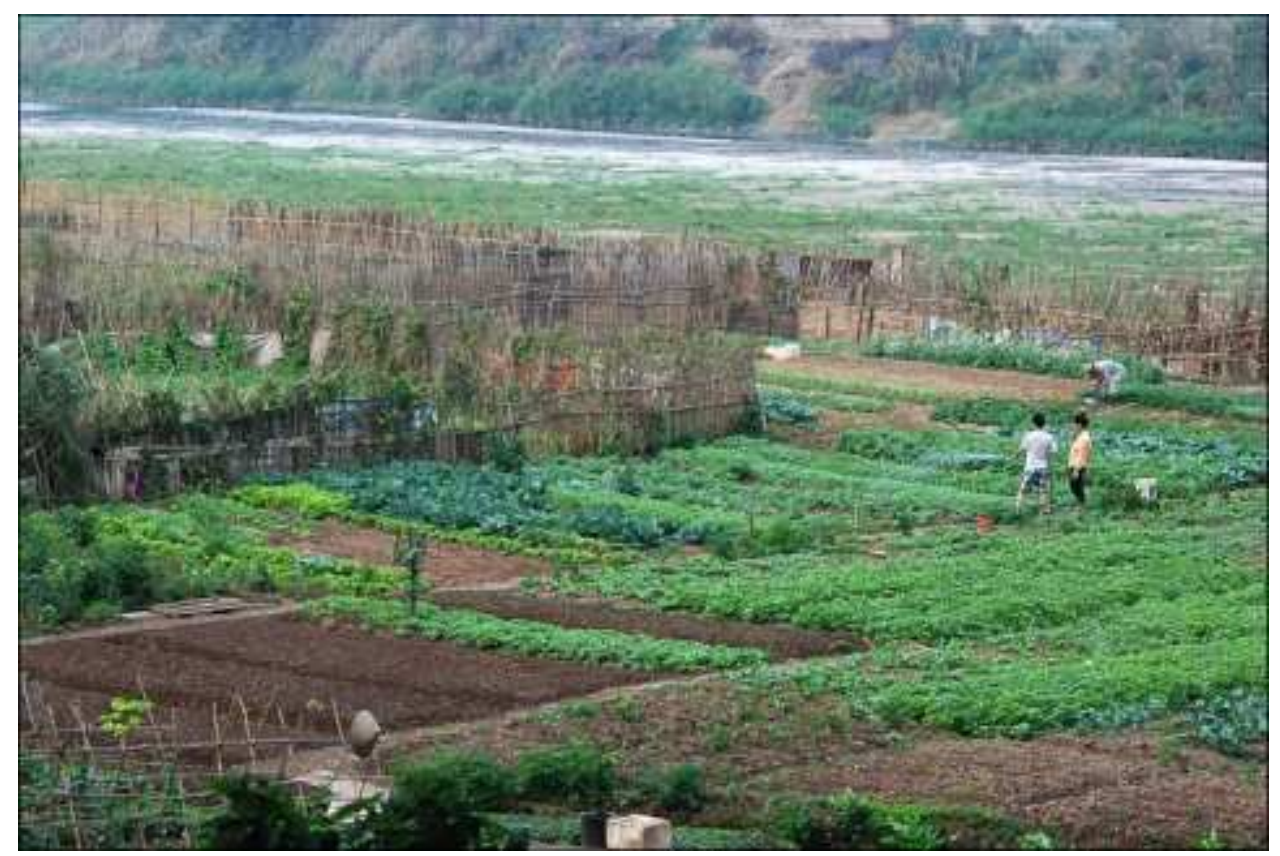

Auteur : G. Pulliat, 2011.

\section{Illustration $\mathbf{3}$ b}




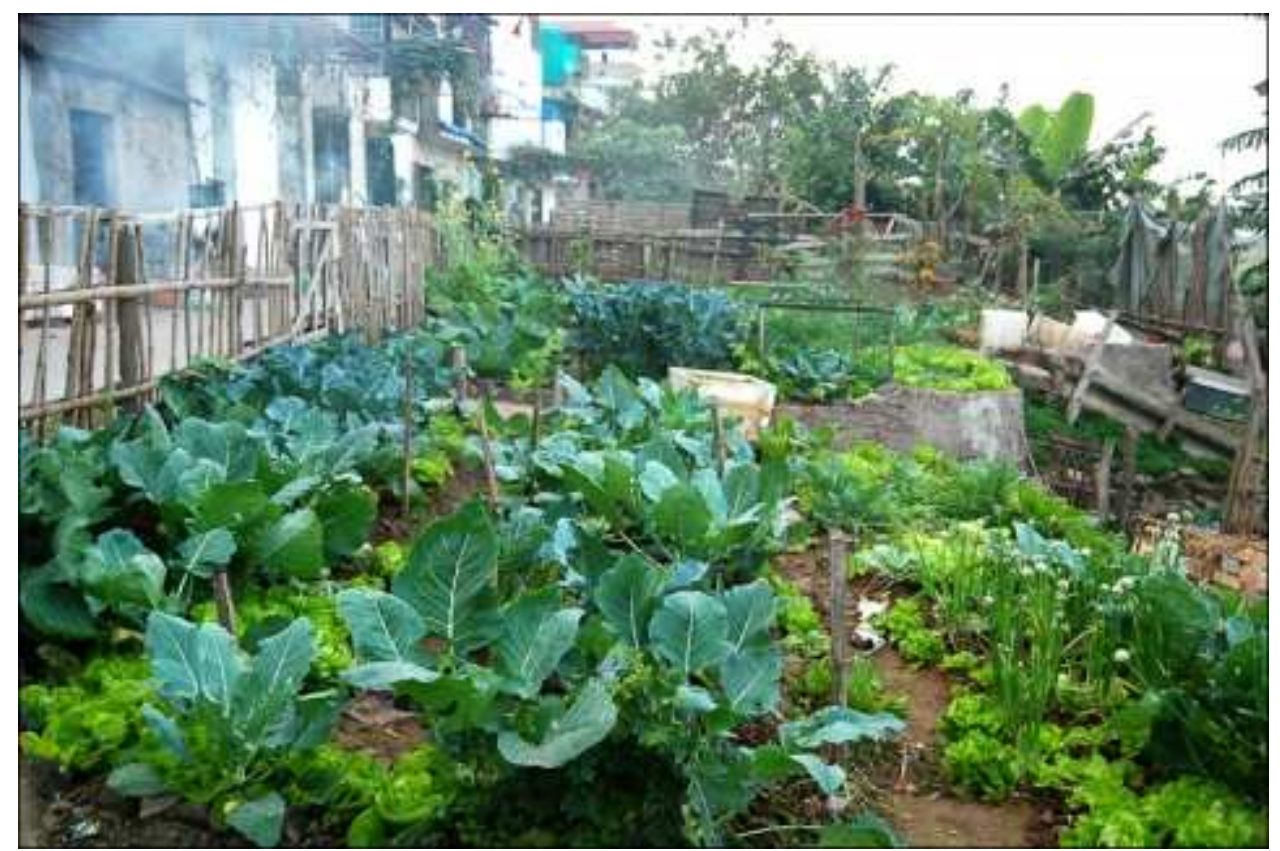

Auteur : G. Pulliat, 2011.

La fonction de complément économique à cette production familiale est nettement apparue après la crise de 2008 : c'est en réponse à l'inflation moyenne de $30 \%$ des denrées alimentaires (pour une inflation générale de l'ordre de $20 \%$ sur l'année) que la mise en culture de la berge du Fleuve Rouge s'est accélérée. Certains riverains (en particulier des personnes âgées n'ayant plus d'activité économique) ont vu là une occasion de s'affranchir partiellement du marché et, dans certains cas, de créer une nouvelle ressource économique en vendant aux voisins les produits de leurs potagers ${ }^{8}$. On assiste ici à une appropriation informelle de l'espace disponible mais non urbanisable qui se présente comme une stratégie de diversification des ressources, tout en répondant à l'attachement à la terre de personnes qui ont longtemps produit elles-mêmes ce qu'elles consommaient, ou qui ont vu leurs ascendants le faire.

Dans ces conditions, on comprend les tensions que créent les expropriations massives des terres agricoles familiales dans la périphérie de la capitale (c'est le cas par exemple de l'ensemble des terres agricoles du village périurbain enquêté) : le recul de la production familiale est perçu comme une perte de sécurité dans l'approvisionnement alimentaire - ce qui est souligné en particulier par nos enquêtés âgés et retraités. On voit ici se dessiner le hiatus entre d'une part l'urbanisation et la forme que prend l'approvisionnement alimentaire de la ville, et d'autre part le vécu de l'espace et les pratiques quotidiennes d'une partie des citadins et des habitants des zones périphériques, qui se perçoivent de plus en plus vulnérables car dépendants de plus en plus du marché alimentaire avec une réduction progressive des ressources habituellement mobilisées.

L'émergence économique du pays se traduit par l'entrée de produits internationaux sur le marché vietnamien et un intense mouvement d'urbanisation. Elle s'accompagne donc d'une diversification de l'origine des denrées alimentaires : Hanoi combine ainsi un approvisionnement international, des produits en provenance de tout le pays, une production de produits frais et périssable dans le Grand Hanoi et les régions limitrophes, et enfin la persistance d'une petite production familiale.

\section{Le marché alimentaire urbain : une recomposition des pratiques}




\section{quotidiennes}

\section{Le développement des supermarchés : un symbole de l'émergence de la ville}

Le projet urbain de Hanoi, tel que défini par le schéma directeur à l'horizon 2030 entend donner à la capitale la forme d'une véritable métropole, avec un centre urbain modernisé, de nouveaux quartiers d'affaires, des zones industrielles associées aux grandes infrastructures de transport, une ceinture verte à vocation maraîchère et récréative, et des pôles urbains périphériques nouveaux. Dans la ville centre, les nouveaux quartiers en construction et les zones en rénovation font la part belle aux tours (pour le logement comme pour les bureaux), aux grandes artères de circulation dessinant de grands îlots urbains, associés à d'importants centres commerciaux : on voit là l'influence du modèle des grandes capitales de la région, telles Séoul ou Bangkok. Ce modèle urbain que l'on voit apparaître dans les nouveaux quartiers, qui colle à l'image de la ville émergente, est associé à un symbole de la modernisation de la vente en ville : il s'agit des supermarchés. Inexistants à Hanoi jusqu'en 1990, ils étaient déjà au nombre de 32 dix ans plus tard (Moustier, Dao, 2006) et de 110 à la fin de l'année 2011 (Courrier du Vietnam, 17/05/2012) : leur croissance est extrêmement rapide, même s'il s'agit souvent de petites surfaces et que tous n'ont pas une vocation alimentaire.

Les supermarchés alimentaires assurent notamment la vente de produits d'épicerie et surtout de produits agro-industriels : confiserie, huiles, pâtes instantanées, produits laitiers, additifs alimentaires (glutamate par exemple)... Il s'agit notamment de produits associés par les consommateurs à une image de modernité, qui s'adressent à une clientèle issue des nouvelles classes citadines moyennes et supérieures qui ont largement entamé la transition de leur régime alimentaire (Charvet, 2009), transformations qui seront précisées dans la troisième partie de cet article. Moustier et Dao (2006) observent ainsi que la population urbaine défavorisée a une opinion positive de ces lieux de vente mais ne les fréquente guère : $60 \%$ de leurs enquêtés, qui ont tous un revenu inférieur au seuil de pauvreté, n'y ont jamais rien acheté et moins de $3 \%$ y font régulièrement des achats. De la même façon, aucun de nos enquêtés ne s'approvisionne en supermarché. Il s'agit bien là d'un symbole d'un mode de vie urbain moderne mais qui accroît les contrastes entre les pratiques quotidiennes des différentes couches sociales urbaines.

\section{La persistance d'un approvisionnement dans la rue à contrecourant de la planification urbaine}

En dépit de leur essor, les supermarchés demeurent pour l'instant quatre fois moins nombreux que les marchés traditionnels (Courrier du Vietnam, 17/05/2012), et tous ne vendent pas de produits frais. En effet, les citadins continuent de majoritairement faire leurs courses dans les marchés, formels ou informels, ou auprès des vendeurs de rue (Moustier, Dao, 2006). Le principal critère de choix du lieu d'approvisionnement reste la proximité (Wertheim-Heck et al., 2011) : ce sont les marchés et les petits commerces proches du domicile ou du lieu de travail, où l'on fait des achats fréquents (parfois plusieurs fois par jour), qui fournissent la majorité des biens alimentaires des Hanoiens, et surtout les produits frais. La connaissance des vendeurs, la facilité d'accès et la possibilité fréquente de faire ses courses sans descendre de moto ou de vélo expliquent largement ces pratiques. 


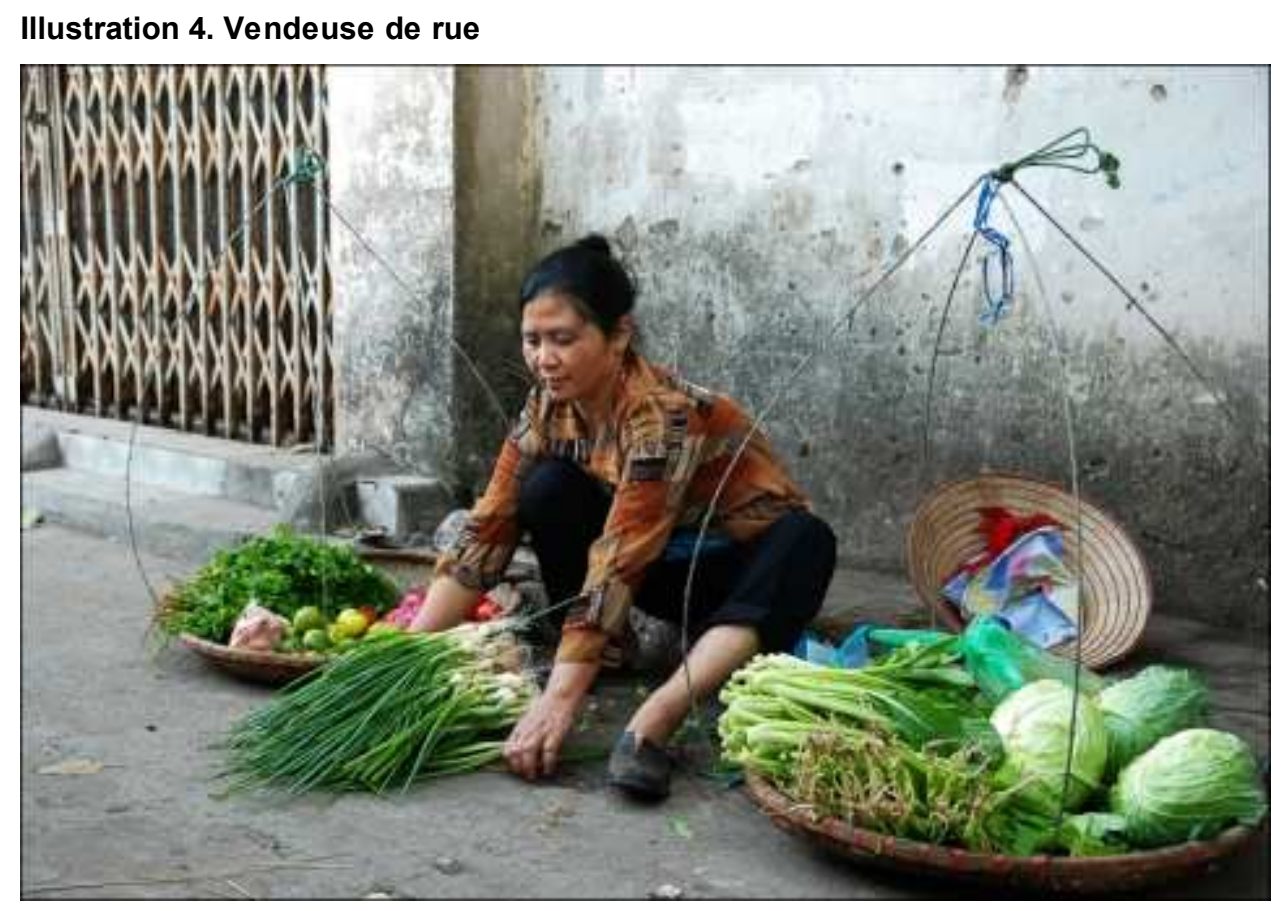

Cette marchande de légumes alterne vente ambulante et vente fixe dans une ruelle du quartier Phuc Tan, près du centre de Hanoi.

Auteur : G. Pulliat, 2011.

Ces achats dans la rue vont à contrecourant des choix politiques du Comité Populaire de Hanoi et du gouvernement vietnamien, décisionnaires de la planification urbaine de Hanoi. Parallèlement au développement des supermarché, une modernisation des marchés de produits frais est en cours, en supprimant certains marchés informels dans la rue et en transformant les infrastructures des marchés formels afin de les doter de zones réfrigérées, de systèmes d'évacuation des eaux usées, d'un service amélioré de gestion des ordures etc. L'objectif exprimé est d'améliorer les conditions d'hygiène, mais aussi de diversifier les produits vendus pour en faire des zones de chalandise plus structurées. L'exemple du marché de Hang Da, en plein centre de la capitale, illustre ces choix (Geertman, 2011) : le marché alimentaire a été relégué au sous-sol, tandis que le rez-de-chaussée et les étages accueillent une galerie marchande. Les clients sont donc contraints de garer leur véhicule (et de payer le parking), de descendre jusqu'au sous-sol pour la partie alimentaire, et n'ont le choix qu'entre un petit nombre d'étals (par exemple, il n'y a que deux marchands de fruits). En contrepartie, des réfrigérateurs permettent de conserver les produits frais - en particulier la viande - au froid, ce qui présente indéniablement un avantage par rapport à leur conservation toute la journée durant, à température ambiante, dans la rue. Mais finalement, ce sont en réalité les nombreux petits étals de produits frais apparus dans les rues adjacentes qui ont pris le relai de l'ancien marché. La politique de rénovation des marchés urbains se fonde pourtant sur ce type de modèle (Courrier du Vietnam, 17/05/2012) et sur la disparition des marchés dans la rue : on voit ici le contraste entre le projet urbain de Hanoi et la structuration de l'espace générée par les usages des citadins.

Illustration 5 - Projet de recomposition de la distribution alimentaire à Hanoi : vers une disparition des petits marchés informels et une rénovation des grands marchés officiels 


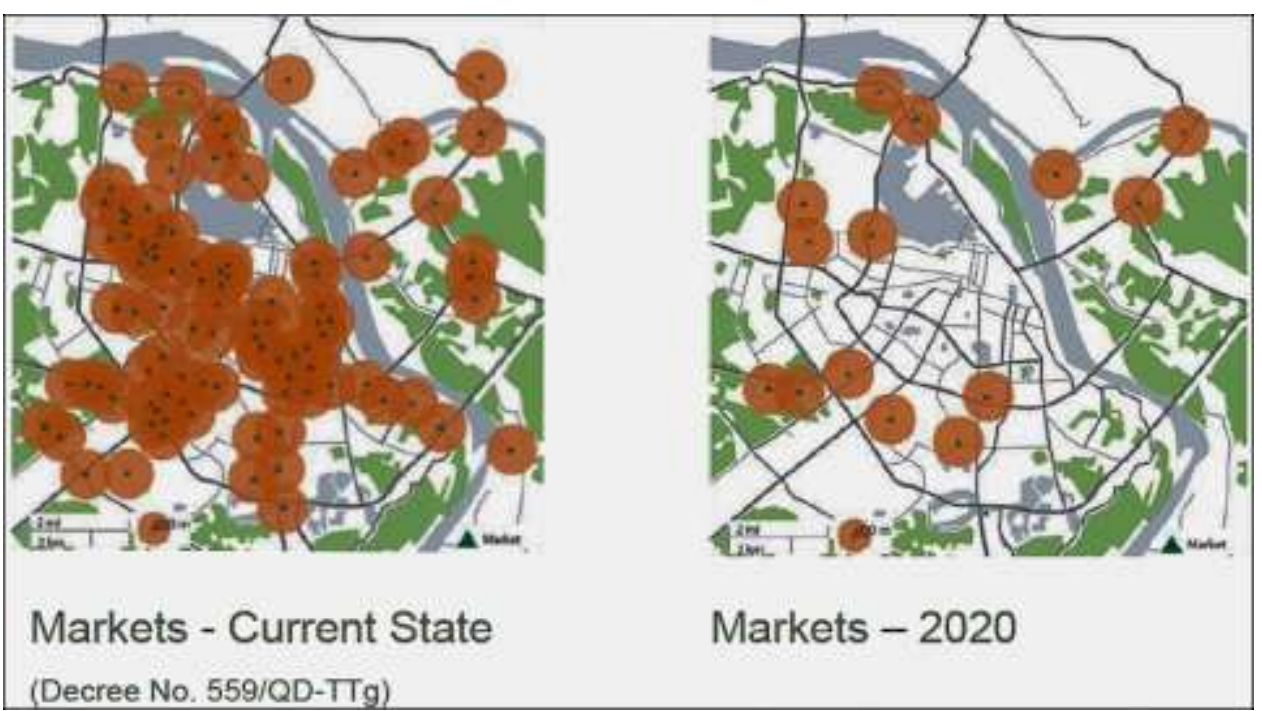

Le modèle posé est une distribution alimentaire assurée par quelques grands marchés et par des magasins formels en dur (supermarchés, épiceries etc.), prenant le relai des petits marchés et de la vente de rue.

Source : Greetman , 2011.

De la même façon, une loi interdisant la vente ambulante a été votée en 2008. En pratique, les vendeurs de rue se voient parfois condamnés à payer de petites amendes, sans confiscation de leurs produits, et ce en particulier s'ils sont susceptibles de gêner la circulation - ce qui dépend de l'appréciation de la police. Pourtant, la vente ambulante assure une desserte des ruelles, encore très présentes dans la morphologie hanoïenne, où les boutiques sont peu nombreuses. Cela assure notamment l'approvisionnement de personnes peu mobiles - au premier rang desquelles les personnes âgées.

Ces choix politiques de «formalisation » du marché et de la vente s'inscrivent dans la stratégie d'image de la ville développée par les autorités locales : la vente de rue sied mal à l'image de la ville émergente (Anjaria, 2006 ; Monnet, 2006 ; Cabral Gomes, Réginensi, 2007). Il n'en demeure pas moins que cette forme d'approvisionnement dans la rue reste ancrée dans les habitudes des Hanoiens, surtout parce qu'elle a pour attribut d'être locale. Cette proximité assure le tissage de relations de confiance entre vendeurs et acheteurs : ces derniers peuvent ainsi réaliser, quand nécessaire, des achats à crédit (Moustier et al., 2006). De plus, une bonne relation commerciale assure de payer le meilleur prix possible sans avoir à marchander longuement. Enfin, ils estiment alors avoir une meilleure connaissance de l'origine des produits et transposent aux denrées la confiance qu'ils font au vendeur.

\section{L'émergence d'une préoccupation concernant la qualité sanitaire des aliments}

Les consommateurs comme les pouvoirs publics se révèlent très préoccupés par la qualité sanitaire des aliments vendus en ville. Du côté des pouvoirs publics, l'image d'une ville moderne est d'abord celle d'une ville salubre, et la stratégie de transformation des lieux d'approvisionnement aux dépens de la vente de rue répond d'abord à la volonté d'améliorer les conditions d'hygiène. Cela s'accompagne de campagnes d'information à destination des consommateurs sur l'importance de laver proprement les produits frais et sur leur conservation par exemple, mais aussi à destination des commerces alimentaires (vendeurs et petits restaurateurs), notamment par le biais de l'Union des Femmes et de la Croix Rouge (associations parapubliques) qui leur fournissent des indications sur les normes d'hygiène et délivrent des certificats aux commerçants qui respectent les critères de leur charte 
(définis par le Ministère de la Santé).

Du côté des consommateurs, l'émergence de la préoccupation concernant la qualité sanitaire des aliments ne prend pas le même sens : elle porte sur les résidus de pesticides et plus généralement sur les produits chimiques utilisés dans la production alimentaire, bien plus que sur les questions d'hygiène. En effet, de nombreux reportages dans les médias comme des campagnes publiques d'information insistent sur la présence de ces résidus dans de nombreux aliments, sur leur nocivité, en particulier quand, par exemple, sont utilisés des pesticides interdits au Vietnam mais illégalement importés de Chine (Wertheim-Heck et al., 2011). Une étude récente (Pham Van Hoi, 2009) a conclu que les deux tiers environ des légumes du marché sur lequel portait l'étude ne répondaient pas aux normes sanitaires et présentaient des taux trop élevés de produits phytosanitaires. Ces informations génèrent une crainte chez l'ensemble des consommateurs, tant dans les milieux favorisés que parmi nos enquêtés aux faibles revenus, sans que des solutions fiables s'offrent à eux en l'absence de traçabilité suffisante et de labellisation fiable. De surcroit, si cette crainte des produits phytosanitaires porte surtout sur les produits frais, à commencer par les fruits, les différents scandales sanitaires dans la région (comme le retentissant cas chinois du lait en poudre pour bébé et des produits laitiers avec de la mélamine en $2008^{9}$ ) transposent également cette peur sur le terrain des aliments agro-industriels.

$\mathrm{Au}$ total, les formes de développement que prend la ville émergente conduisent à une recomposition de la distribution alimentaire dans la ville. On observe d'un côté une modernisation des lieux de vente avec l'essor des supermarchés et la transformation des marchés traditionnels, mais ceux-ci, plus onéreux que la vente de rue, sont surtout fréquentés par une population urbaine plutôt favorisée qui associe ces lieux de vente à une image de meilleure sécurité sanitaire (Moustier, Dao, 2006) et de produits modernes. D'un autre côté, on observe la persistance d'un approvisionnement quotidien dans la rue et dans les marchés traditionnels, auxquels sont associées l'image de fraîcheur des produits mais aussi la crainte des produits phytosanitaires. L'émergence de la ville se traduit par deux phénomènes dans la distribution alimentaire : d'une part l'accroissement des contrastes dans les modes d'approvisionnement selon les couches sociales, d'autre part l'émergence de la question sanitaire comme sujet majeur de préoccupation - à l'image de ce qui se passe en Chine par exemple.

\section{La consommation : transition alimentaire, vulnérabilité et inégalités}

\section{Entre transition alimentaire engagée et insécurité alimentaire persistante, des inégalités croissantes selon les revenus}

Jean-Paul Charvet (2009) définit la transition alimentaire comme le passage d'un régime alimentaire centré sur les céréales à un régime plus diversifié, incluant une plus grande part de produits carnés et piscicoles, de matière grasse, de fruits et légumes et de produits laitiers. Cette transition s'effectue sous l'effet de l'augmentation du niveau de vie et apparaît généralement en ville avant de se diffuser aux espaces ruraux environnants (Landy, 2008). La très rapide réduction de la pauvreté qu'a connue le Vietnam, en particulier en ville ${ }^{10}$, s'est accompagnée de profondes transformations dans le régime alimentaire des citadins. En ville, entre 1989 et 2000, la consommation de riz est passée de $154 \mathrm{~kg}$ par an et par personne à 
$123 \mathrm{~kg}$ (Calandre, 2006), cependant qu'augmentait la consommation de blé (notamment sous forme de nouilles instantanées et de pain). Entre le Đối Mói et les années 2000, à Hanoi, la consommation de viande a triplé, la consommation de produits laitiers a quadruplé (de 2 à $9 \mathrm{~kg}$ par an et par personne), la consommation de fruits a décuplé, pendant que les friandises, les biscuits, les aliments industriels transformés (dont les saucisses, les nouilles instantanées, les auxiliaires de cuisine comme les bouillons-cube et le glutamate monosodique) ont fait leur apparition (Calandre, 2006). La capitale a suivi le modèle classique de la transition alimentaire, à une vitesse remarquable.

Cependant, notre enquête montre que, au sein de la population urbaine à faible revenu, on continue d'observer un régime alimentaire centré sur des aliments de base, peu diversifié, et parfois insuffisant en termes de quantité. Nous avons interrogé nos enquêtés sur leurs habitudes alimentaires, la fréquence de la consommation des différents produits, en partant d'un rappel des consommations de la journée précédente puis en les interrogeant plus longuement sur les pratiques des dernières semaines écoulées. Si notre approche qualitative ne permet pas de donner une évaluation de la qualité nutritionnelle de leur alimentation, elle donne à voir les grandes lignes des habitudes alimentaires quotidiennes et les différences importantes qui existent dans ces pratiques selon les revenus et les contraintes budgétaires.

Ainsi, on a dessiné trois grands types de comportements alimentaires quotidiens :

- Un premier groupe constitué des personnes qui, tout en accordant une part importante de leurs revenus à l'alimentation (plus de 60\%), ont un budget alimentaire très réduit. Elles ne prennent que deux repas par jour (mais les enfants de la famille, eux, consomment au moins du riz blanc pour le petitdéjeuner), ne consomment de viande, poisson, œuf ou tofu qu'une fois par jour au maximum, et il s'agit le plus souvent du tofu, parce que très bon marché. Le repas de base est constitué de riz blanc, de légumes-feuilles (liserons d'eau le plus souvent) et de bouillon, tandis que les autres types de légumes sont consommés seulement de façon occasionnelle. On observe ici des situations d'insécurité alimentaire, caractérisée par la faiblesse des prises alimentaires tant en termes de quantité que de diversité - ce qui grève la qualité nutritionnelle. Cela représente environ un quart de nos enquêtés.

- Un second groupe n'est pas en insécurité alimentaire : le petit déjeuner, même frugal, devient systématique ; le plat de viande/poisson/œuf/tofu (une à deux fois par jour) comme les légumes consommés varie chaque jour. L'alimentation constitue une part toujours importante du budget (généralement de l'ordre de la moitié) ; le budget quotidien permet d'assurer une alimentation suffisante et diversifiée, mais fondée seulement sur des produits de base. L'achat de fruits (relativement onéreux), de produits laitiers et de friandises reste occasionnel. Ce groupe représente environ la moitié des enquêtés.

- Le troisième groupe a des pratiques témoignant de la transition alimentaire engagée. Un plat de viande (y compris autre que du porc), d'œufs ou de poisson est présenté à tous les repas, et le choix se fait plus en fonction des préférences que des coûts. Les achats de produits laitiers pour les enfants deviennent réguliers (au moins une fois par semaine, et quotidiennement pour les enfants de moins de 2 ans), de même que la consommation de fruits et de friandises, biscuits etc. On observe aussi la consommation d'autres féculents que du riz blanc : des pates de riz fraîches, des nouilles au blé et aux œufs par exemple. Ce groupe représente environ un tiers de nos enquêtés. quotidiennes, selon le niveau de revenu. La transition alimentaire n'est pas achevée 
à Hanoi, et les personnes à faible revenu continuent à se trouver parfois en situation d'insécurité alimentaire.

\section{La transition nutritionnelle : sur les traces du modèle chinois ?}

32 Cette différenciation croissante se traduit également par la coexistence, à Hanoi, de situation de malnutrition par carences et de troubles liés à la suralimentation : c'est ce que N. Calandre (2006) nomme la transition nutritionnelle. D'un côté, la malnutrition a beaucoup diminué : elle a baissé de plus de $25 \%$ entre 1985 et 2002 (Calandre, 2006). Selon l'Unicef, à l'échelle du pays près d'un tiers des enfants seraient en situation de malnutrition ; si cela concerne d'abord les enfants des zones rurales éloignées des deltas, surtout parmi des minorités ethniques, la malnutrition par carences continue à se rencontrer à Hanoi, parmi les enfants (surtout le groupe 6-24 mois) mais aussi parmi les adultes (NIN, 2011).

D'un autre côté, alors qu'inexistants avant 1985, le surpoids et l'obésité ont fait leur apparition en ville, même si leur ampleur reste limitée : ils concernent 7,6 \% des urbains aujourd'hui (contre $3,3 \%$ des ruraux seulement). Cela devient néanmoins un sujet de préoccupation car on peut s'attendre à une croissance rapide de ce phénomène : ainsi, à Hanoi près de $11 \%$ des filles de 6 à 11 ans sont en situation de surpoids ou d'obésité (Calandre, 2006). C'est un enjeu de santé publique en devenir, particulièrement préoccupant quand on observe la situation du voisin chinois, qui connait aujourd'hui des taux d'obésité remarquables, en particulier parmi les enfants citadins. Un article de la chaîne nationale CCTV (2010) révèle que le taux d'enfants d'âge scolaire en surpoids et obèses en ville est passé de $2 \%$ en 1988 à plus de $16 \%$ aujourd'hui, ce qui s'explique par les transformations alimentaires avec un accroissement de la part des graisses et des aliments sucrés dans le régime des citadins, et par les changements de modes de vie réduisant l'activité physique. Les mutations en cours au Vietnam laissent à penser que le pays pourrait suivre le chemin de son voisin.

\section{Vulnérabilité alimentaire et résilience}

Notre enquête a mis au jour l'existence, à Hanoi, de situations de vulnérabilité alimentaire : c'est-à-dire une capacité relativement faible à endurer des stress de toute nature (Janin, 2006) conduisant un sujet à se retrouver de façon transitoire ou durable en situation d'insécurité alimentaire. Face à un choc (baisse des revenus, frais de santé à payer etc.), les familles vulnérables se voient contraintes de réduire leur budget alimentaire et se retrouvent en insécurité.

35 Le premier facteur de vulnérabilité se trouve être la part importante de l'alimentation dans le budget familial. Selon l'enquête nationale sur les conditions de vie des ménages (VHLSS, 2010), l'alimentation (et les boissons) représente $49 \%$ des dépenses des ménages, et ce taux atteint $65,4 \%$ dans le premier quintile des revenus (ce qui est stable depuis le début des années 2000), contre seulement $44,8 \%$ dans le cinquième quintile (avec une tendance à la baisse). C'est comparable à la situation indienne, où $43 \%$ du revenu disponible des urbains sont attribués à l'alimentation, mais bien plus important qu'en Chine où seulement $26 \%$ du budget $y$ sont consacrés (Landy, 2008). Ce taux élevé induit une grande vulnérabilité face aux fluctuations des revenus (qui s'explique par la précarité des formes d'emploi) qui se traduit parfois par des périodes d'insécurité alimentaire. Cette vulnérabilité est accrue par l'inflation alimentaire, qui, depuis le début des années 2000, est systématiquement plus élevée que l'inflation générale. Elle a été particulièrement 
forte en 2008 et en 2010, de façon symétrique aux marchés mondiaux des denrées alimentaires.

36 Quelles sont alors les stratégies mises en place par les ménages pour faire face aux périodes de difficulté ? Il convient d'abord de souligner le manque d'efficacité de l'aide publique face à ces difficultés transitoires. L'aide publique prend deux formes : celles d'une sécurité sociale, créée en 1947, qui ne concerne que les salariés qui cotisent ${ }^{11}$, et d'une aide sociale qui est accordée par les autorités locales et les associations para-étatiques (en particulier l'Union des Femmes). Cette dernière se présente soit sous forme de prêts à taux d'intérêts réduits ou nuls, soit sous forme de statut de pauvre ouvrant droit à une assurance santé gratuite et à une réduction des frais de scolarité. Les personnes les plus âgées (plus de 80 ans) et les handicapés peuvent également bénéficier d'une allocation mensuelle (un peu inférieure au seuil de pauvreté). Mais l'obtention de ces aides prend du temps souvent plusieurs mois -, de sorte qu'elles ne représentent pas une ressource pour faire face aux difficultés transitoires.

Au quotidien, donc, c'est à l'aide des proches que l'on recourt. On emprunte de petites sommes pour faire les courses, que l'on rembourse quelques jours plus tard quand les revenus le permettent; ou bien des sommes plus importantes qui constituent une dette que l'on rembourse ultérieurement - sans intérêts - au gré des possibilités. Les entretiens réalisés ont montré que l'emprunt de petites sommes à des voisins ou des parents est la première ressource mobilisée pour faire face à un manque d'argent, bien avant l'achat à crédit. On observe ainsi une intense circulation d'argent entre les individus, qui assure aux plus pauvres la possibilité de lisser leurs dépenses, et, partant, de limiter les préjudices alimentaires de revenus fluctuants ou d'écarts importants entre revenus et dépenses.

Ces échanges de court terme s'appuient sur l'entretien des réseaux de solidarité dans le long terme. Le bénéfice de cette aide n'est pas sans coût : il implique de participer aux cérémonies des voisins et des proches (mariages, funérailles, etc.), sous forme d'un don en numéraire, mais aussi de donner (en numéraire ou en nature) quand ces derniers rencontrent des difficultés. Il y a donc un principe de réciprocité dans le long terme, même si le contre-don n'est pas nécessairement équivalent au don. Dès lors, la participation aux cérémonies est une priorité budgétaire, y compris pour les personnes les plus pauvres : au cours des mois où les cérémonies sont particulièrement nombreuses ${ }^{12}$, ils n'hésitent pas à réduire leur budget alimentaire afin d'être en mesure de participer. Ils peuvent alors se trouver en insécurité alimentaire transitoire, mais s'assurent ainsi de pouvoir bénéficier d'une aide dans le long terme, et donc d'accroître leur résilience à longue échéance.

Ces réseaux sociaux locaux fondés sur les dons, donc l'endettement mutuel, persistent largement en ville. L'augmentation du niveau de vie et l'obtention d'emplois stables et de revenus réguliers s'accompagnent évidemment d'une diversification des ressources en cas de difficultés, comme le recours à un crédit classique, mais ces réseaux de solidarité ne se diluent pas avec la formalisation de l'économie ; on observe leur existence à tous les niveaux sociaux.

40 En conclusion, l'analyse de la consommation alimentaire à Hanoi témoigne d'une transition alimentaire en cours, mais qui reste inachevée et concerne encore de façon marginale les groupes à faibles revenus ; il en résulte une différenciation croissante dans les régimes alimentaires adoptés par les citadins. La forte croissance économique à l'origine d'une rapide réduction de la pauvreté en ville a permis d'améliorer considérablement la sécurité alimentaire des ménages, mais demeurent des familles vulnérables qui se trouvent périodiquement ou durablement en difficulté pour se nourrir de façon satisfaisante. Persistent également les réseaux de solidarité anciens, à tous les niveaux sociaux, qui permettent de limiter les dommages alimentaires quand des difficultés économiques surviennent et accroissent la résilience des familles. 


\section{Conclusion}

L'analyse du système alimentaire à Hanoi permet d'illustrer les recompositions survenant dans un contexte qui connaît des transformations rapides. Si l'émergence économique caractérise un pays qui connaît une forte croissance économique sur une longue période et une ouverture croissante de son marché à l'international, son impact dans le domaine alimentaire semble se lire, dans le cas de Hanoi, en termes de contrastes. Contrastes dans l'offre alimentaire d'abord, par sa diversité croissante, tant en termes de produits que d'origine : c'est l'articulation de plusieurs échelles et plusieurs formes de production qui fonde cette diversité. Contrastes dans la distribution ensuite, avec la coexistence d'un réseau formalisé, à l'image du réseau d'approvisionnement occidental, qui répond à la volonté de l'État de dessiner une ville moderne, et d'un réseau d'approvisionnement informel, qui répond aux usages des citadins (courses quotidiennes, de proximité, surtout pour les produits frais), en particulier les moins aisés qui n’ont pas accès au secteur modernisé, cependant que l'achat en supermarché est une pratique valorisée par la classe moyenne émergente. A cet égard, Hanoi semble encore éloignée du modèle chinois où le commerce formel représente déjà $30 \%$ de l'ensemble du commerce de détail (Landy, 2008). Contraste dans la consommation enfin, avec une transition alimentaire engagée mais qui concerne surtout les citadins les plus favorisés, symbolisée par la coexistence d'insécurité alimentaire, de malnutrition par carences et l'apparition des problèmes de surpoids.

Ainsi, l'accroissement des inégalités que connait le Vietnam depuis le début des années 2000 se lit dans les usages alimentaires. On voit une recomposition progressive des pratiques en cours, avec une adaptation des habitudes quotidiennes selon les contraintes et les opportunités de chacun. Les transformations dont la ville fait l'objet (étalement urbain, projets de modernisation) pèsent sur certains types de ressources des urbains défavorisés (terres agricoles, commerce de rue peu onéreux), les laissant plus vulnérables face aux nouveaux enjeux dont l'inflation alimentaire, sans que cela semble pris en compte par les pouvoirs publics. En définitive, seule la persistance des solidarités permet aux citadins les plus pauvres de ne pas connaître d'aggravation globale de leur situation alimentaire.

\section{Bibliographie}

Anjaria J.S., 2006. Street Hawkers and Public Space in Mumbai. Economic and Political Weekly, vol. $41 \mathrm{n}^{\circ} 21, \mathrm{p} 2140-2146$.

Bricas N., Abdoulaye Seck P., 2004. L'alimentation des villes du Sud : les raisons de craindre et d'espérer. Cahiers Agricultures, vol. 13, ${ }^{\circ}{ }^{\circ}$, John Libbey Eurotex, p. 10-14.

Bricas N., Dury S., Figuié M., Maire B., Delpeuch F., 2003. Sécurité alimentaire et urbanisation ; enjeux pour l'agriculture intra et péri-urbaine. Séance de l'Académie d'Agriculture : Approvisionnement alimentaire et production agricole de proximité pour les villes du Sud : enjeux de l'agriculture urbaine et péri-urbaine, http://www.academie-agriculture.fr /mediatheque/seances/2003/20031217communication3_integral.pdf

Cabral Gomes M.F., Réginensi C., 2007. Vendeurs ambulants à Rio de Janeiro : expériences citadines et défis des pratiques urbaines. Cybergeo : European Journal of Geography [En ligne], http://cybergeo.revues.org/4870 (consulté en déc. 2011).

Calandre N., 2006. Pratiques et perception des risques nutritionnels : Les mères face aux malnutritions infantiles au Vietnam. Thèse de doctorat, Université de Montpellier ;

Cao X.D, Tran T.-A.D, 2005. Transition et ouverture économique au Vietnam : une différenciation sectorielle. Economie internationale 4/2005, no104, p. 27-43, www.cairn.info/revue-economie-internationale-2005-4-page-27.htm

Castiglioni F., Cusset JM., Gubry P., Nguyên T.T., Pham T.H. (dir.), 2006. La ville vietnamienne en transition. Paris, Karthala, IMV, PADDI, $314 \mathrm{p}$.

CCTV, 29/01/2010. L'obésité chez les jeunes en Chine. http://fr.cctv.com/program 
/objectifchine/20100129/101868.shtml

Charvet J., 2009. L'alimentation dans le monde. Mieux nourrir la planète. Paris, Larousse, $128 \mathrm{p}$.

Cling J., Marouani M.A., Razafindrakoto M., Robilliard A., Roubaud F., 2008. The distributive impact of Vietnam's accession to the WTO. Economie Internationale/International Economics, $\mathrm{n}^{\circ} 118$, p. 43-71.

Courrier du Vietnam, 25/01/2011. Vers une diversification du système de protection sociale 2011-2020.http://lecourrier.vnagency.com.vn/default.asp?xt=\&ct=\&page=newsdetail\& newsid $=69340$

Courrier du Vietnam, 17/05/2012. Les marches alimentaires repoussés en banlieue. http://lecourrier.vnanet.vn/lecourrier/fr-fr/details/5o/vie/47540/les-marches-de-hanoirepousses-en-banlieue.aspx

Dao The Tuan, 2002. Vietnam : Réformes agraires successives et succès de l'agriculture familiale, in Merlet, M., Politiques foncières et réformes agraires. Paris, IRAM.

Fanchette S., Dao The Anh, 2008. La crise alimentaire mondiale : une opportunité pour relancer la filière rizicole au Vietnam ? Hérodote, $\mathrm{n}^{\circ}{ }^{131}$, Paris, La Découverte, p. 175-193.

FAO, 2005. La situation dans le monde de l'agriculture et de l'alimentation : Le commerce agricole et la pauvreté. Rome, FAO, $225 \mathrm{p}$.

Figuié M., 2004. Perception of food-related risks by consumers in Hanoi. Vietnam. CIRAD/IOS, MALICA, Hanoi, 18 p.

Geertman S., 2011. Urban Transition and Food Environment in Hanoi, Vietman. Communication at The Third Engaging with Vietnam: an Interdisciplinary Dialogue, Conference, Hanoi, $5^{\text {th }}$ dec. 2011

Gironde C., 2009. Réformes, croissance, et augmentation des inégalités dans le delta du fleuve Rouge - Viêt Nam (1980-200o). Moussons, ${ }^{\circ} 13-14$, p. 269-308

GSO (General Statistics Office), http://www.gso.gov.vn/default_en.aspx?tabid=491

Jaffrelot C. (dir.), 2008. L'enjeu mondial : Les pays émergents. Paris, Presses de Sciences Po, $381 \mathrm{p}$.

Janin P., 2006. La vulnérabilité alimentaire des Sahéliens : concepts, échelles et enseignements d'une recherche de terrain. Espace Géographique, tome 35, p. 355-366.

JICA (Japanese International Cooperation Agency), 2007. The Comprehensive Urban Development Program in Hanoi Capital City of the Socialist Republic of Vietnam (HAIDEP). Final report, $569 \mathrm{p}$

Landy F., 2008. Nourrir 2,5 milliards de personnes, plus et mieux : les défis de l'Inde et la Chine, in Charvet J.P., Nourrir les hommes, SEDES, p. 184-224.

Malassis L., 1993. Nourrir les hommes, Paris, Flammarion, 128 p.

Maxwell S., 1996. Food security: a post-modern perspective. Food Policy, vol.21 nº 2, p155-170

Maxwell S., Smith M., 1992. Household food security: a conceptual review, in Maxwell S., Frankenberger T.R. (ed.), Household Food Security: Concepts, Indicators, Measurements: A Technical Review, New York, Rome, UNICEF et IFAD.

Monnet J., 2006. L'ambulantage : Représentations du commerce ambulant ou informel et métropolisation. Cybergeo : European Journal of Geography [En ligne], http://cybergeo.revues.org/2683 (consulté en déc. 2011).

Moustier P., Dao The Anh et al., 2006. Supermarkets and the poor in Vietnam. Hanoi, Malica.

Moustier P., Vagneron I., Bui T.T., 2004. Organisation et efficience des marchés de légumes approvisionnant Hanoi (Vietnam). Cahiers Agricultures, vol. 13, $\mathrm{n}^{\circ}$ 1, John Libbey Eurotex, p. $142-147$.

NIN (National Institute of Nutrition), 2011. Summary report general: Nutrition survey 2009-201O. Hanoi, Ministry of Health et UNICEF, 14 p.

Pham Thai Son, 2010. Morphologie urbaine, dispositifs techniques et pratiques sociales : cas des quartiers de ruelles hanoiens. Thèse de doctorat, Institut National des Sciences Appliquées de Lyon.

Pham Van Hoi et al., 2009. Market governance for safe food in developing countries: The case of low-pesticide vegetables in Vietnam. Journal of Environmental Management, $\mathrm{n}^{\circ}$ 91, p. $380-388$.

Poverty Task Force, 2002. Localizing MDGs for poverty reduction in Viet Nam : Reducing vulnerability and providing social protection. Hanoi, Banque Mondiale.

Poverty Task Force, 2005. Regional Poverty Assessment: Red River Delta region. Hanoi, Banque Mondiale. 
Rossi G. (dir), 2002. Périurbanisation de la province d'Hanoi. Atlas infographique de la province de Hanoi. Hanoi, Maison d'édition de la cartographie, Hamatra, $382 \mathrm{p}$.

Sautier D., Dao The Anh, Pham Cong Nghiep, Nguyen Ngoc Mai, 2011. Agriculture et croissance urbaine à Hanoi. Présentation au ge Forum économique et financier francovietnamien, à venir, Hanoi, Adetef, 19-20 mars 2011.

Van de Berg L.M., Van Wijk M.S., Pham Van Hoi, 2003. The transformation of agriculture and rural life downstream of Hanoi. Environment \& Urbanization, vol. 15, nº 1, p. 35-52.

VHLSS (Vietnam Households Living Standards Survey), 2010. Hanoi, General Statistics Office, http://www.gso.gov.vn/default_en.aspx?tabid=515\&idmid=5\&ItemID=12426

Villes en transition, 2005. Impact of Existing Residence Registration Policy on Urban Poverty Alleviation. Hanoi, Institute of Sociology.

Wertheim-Heck S., Vellema S., Spaargaren G., 2011. Vegetable purchasing in the transitional economy of Vietnam: Investigating the daily practice of food shopping to identify mechanisms of trust in food safety. Hanoi, publication à venir.

\section{Notes}

1 Généralement traduit par " renouveau », le Đổi Mới est un ensemble de politiques de réformes économiques entreprises par l'État vietnamien à partir de 1986 qui ont initié la transition vers une économie de marché : décollectivisation, libéralisation des échanges, ouverture du pays aux investissements étrangers etc.

2 Depuis le début des années 1990, la croissance économique nationale a été en moyenne de $8 \%$ par an (VDR, 2008).

3 Le Viêt-Nam a développé une agriculture typique de la Révolution verte, avec un usage important d'engrais : sa consommation est presque trois fois supérieure à la consommation des pays en développement par hectare $(285 \mathrm{~kg} / \mathrm{ha}$ de terres arables, contre 114 en moyenne dans les pays en développement et 101 dans le monde) (FAO, 2005). Sur les très petites parcelles du delta du Fleuve Rouge, cet usage est très souvent excessif (Moustier, 2004).

4 Selon les années, depuis 1996, le Vietnam est, en volume, le deuxième ou le troisième exportateur mondial de riz, derrière la Thaïlande et au coude à coude avec l'Inde. En valeur, il oscille de la seconde à la quatrième place, avec l'Inde et le Pakistan.

5 Saison de faible production maraîchère dans le delta du Fleuve Rouge.

6 Nous nommons ici « Grand Hanoi » la province de Hanoi selon les limites administratives de 2008 : elle recouvre désormais une superficie de $3345 \mathrm{~km}^{2}$, dont les districts urbains ne recouvrent que $181 \mathrm{~km}^{2}$, et elle accueille une population totale d'environ 6 millions d'habitants, dont seulement 2 millions dans les districts urbains.

7 Qu'il s'agisse des terres obtenues lors de la décollectivisation, d'espaces cultivés dans le jardin de la maison ou encore de petits potagers créés sur les espaces en friches en attendant leur construction (ce qui, dans ce dernier cas, n'est pas toujours pérenne).

8 Les personnes qui mettent la berge en cultures sont généralement celles qui habitent le plus près de l'eau, mais toute personne du quartier a la possibilité, selon les discours des riverains, de défricher un nouvel espace pour créer un jardin. Elles présentent des profils très variés, avec à la fois des personnes à faibles revenus qui ont vu là une ressource supplémentaire et des personnes aisées qui voient là l'occasion de revenir à une production agricole individuelle, ce qui est perçu très positivement.

9 Matière plastique utilisée comme additif afin d'augmenter le taux d'azote, qui est utilisé comme mesure du taux de protéines dans ces produits laitiers ; cela a causé la mort de plusieurs bébés et d'importants problèmes de santé chez de très nombreux consommateurs, probablement de l'ordre de 200 ooo personnes.

10 Le taux de pauvreté selon le seuil officiel (variant d'année en année pour prendre en compte l'inflation) est passé de 58,6 \% en 1993 à 10,6 \% en 2010, cependant que le taux de pauvreté urbaine est passé de 25,1\% à moins de $3 \%$ au cours de la même période (données des recensements du GSO).

11 Soit $14 \%$ de la population active en 2000 (Poverty Task Force, 2002) et $18 \%$ en 2010 selon le Ministère du Travail (Courrier du Vietnam, 25/01/2011).

12 Les mariages en particulier se déroulent majoritairement en automne et en hiver. Les enquêtes ont montré qu'entre octobre et janvier, il n'est pas rare qu'une personne ait à participer à une dizaine de cérémonies dans le mois. Si l'on ne peut pas être présent, on fait parvenir un don d'argent. 


\section{Table des illustrations}

\begin{tabular}{|c|c|c|}
\hline & Titre & $\begin{array}{l}\text { Illustration } 1 \text { - Extension de la tache urbaine dans la province de } \\
\text { Hanoi }\end{array}$ \\
\hline 3 . & Légende & $\begin{array}{l}\text { Le développement urbain de Hanoi a conduit à un étalement de la } \\
\text { ville vers l'ouest principalement, surtout le long des principaux axes } \\
\text { de transport. La ville a progressivement absorbé les anciens villages } \\
\text { périurbains et leurs zones agricoles, qui constituent désormais des } \\
\text { quartiers de la capitale. }\end{array}$ \\
\hline & Crédits & Source : Pham Thai Son (2010), d'après JICA (2007). \\
\hline & URL & http://echogeo.revues.org/docannexe/image/13205/img-1.jpg \\
\hline & Fichier & image/jpeg, 228k \\
\hline & Titre & Illustration 2 - Un village périurbain en transition - An Khánh \\
\hline & Légende & $\begin{array}{l}\text { Le projet de ville nouvelle « Splendora » et « An Khánh Sud » le long } \\
\text { de l'autoroute de l'Ouest s'est accompagné de l'expropriation de } \\
\text { toutes les terres agricoles de la commune, afin de construire de } \\
\text { nouvelles zones d'habitat et un pôle économique. Ici, les rangées de } \\
\text { maisons encore en construction contrastent fortement avec l'habitat } \\
\text { préexistant, qu'il s'agisse des anciennes maisons à un niveau autour } \\
\text { d'une cour, des grosses maisons individuelles ou des maisons-tubes } \\
\text { plébiscitées par les habitants qui reconstruisent leur logement. }\end{array}$ \\
\hline & Crédits & Auteur : G. Pulliat, 2011. \\
\hline & URL & http://echogeo.revues.org/docannexe/image/13205/img-2.jpg \\
\hline & Fichier & image/jpeg, 464k \\
\hline & Titre & $\begin{array}{l}\text { Illustrations } 3 a \text { et } 3 b \text {. Quartier de Phúc Tân, Hanoi : cultures sur la } \\
\text { berge du Fleuve Rouge : chou-rave, laitue, liserons d'eau, chayottes, } \\
\text { plantes aromatiques }\end{array}$ \\
\hline & Crédits & Auteur : G. Pulliat, 2011. \\
\hline & URL & http://echogeo.revues.org/docannexe/image/13205/img-3.jpg \\
\hline & Fichier & image/jpeg, 608k \\
\hline & Titre & Illustration $3 \mathrm{~b}$ \\
\hline & Crédits & Auteur : G. Pulliat, 2011. \\
\hline & URL & http://echogeo.revues.org/docannexe/image/13205/img-4.jpg \\
\hline & Fichier & image/jpeg, 592k \\
\hline & Titre & Illustration 4. Vendeuse de rue \\
\hline & de & $\begin{array}{l}\text { Cette marchande de légumes alterne vente ambulante et vente fixe } \\
\text { dans une ruelle du quartier Phuc Tan, près du centre de Hanoi. }\end{array}$ \\
\hline & Crédits & Auteur : G. Pulliat, 2011. \\
\hline & URL & http://echogeo.revues.org/docannexe/image/13205/img-5.jpg \\
\hline & Fichier & image/jpeg, 500k \\
\hline & Titre & $\begin{array}{l}\text { Illustration } 5 \text { - Projet de recomposition de la distribution alimentaire à } \\
\text { Hanoi : vers une disparition des petits marchés informels et une } \\
\text { rénovation des grands marchés officiels }\end{array}$ \\
\hline 663. & & $\begin{array}{l}\text { Le modèle posé est une distribution alimentaire assurée par quelques } \\
\text { grands marchés et par des magasins formels en dur (supermarchés, } \\
\text { épiceries etc.), prenant le relai des petits marchés et de la vente de } \\
\text { rue. }\end{array}$ \\
\hline & Crédits & Source : Greetman , 2011. \\
\hline & URL & http://echogeo.revues.org/docannexe/image/13205/img-6.jpg \\
\hline & Fichier & image/jpeg, 395k \\
\hline
\end{tabular}

\section{Pour citer cet article}


Gwenn Pulliat, «Se nourrir à Hanoi : les recompositions du système alimentaire d'une ville émergente », EchoGéo [En ligne], numéro 21 | 2012, mis en ligne le 10 octobre 2012, consulté le 01 novembre 2012. URL : http://echogeo.revues.org/13205

\section{Auteur}

Gwenn Pulliat

Gwenn Pulliat, gwenn.pulliat@gmail.com, est doctorante en géographie, Université Paris

Ouest , UMR 7218 LAVUE (CNRS).

\section{Droits d'auteur}

(C) Tous droits réservés 